\title{
The Association between Family Mealtime and Depression in Elderly Koreans
}

\author{
Yunhwa Kang', Soyeon Kang', Kyung Jung Kim', Hyunyoung Ko², Jinyoung Shin ${ }^{3}$, Yun-Mi Song ${ }^{1, *}$ \\ 'Department of Family Medicine, Samsung Medical Center, Sungkyunkwan University School of Medicine, Seoul, Korea \\ ${ }^{2}$ Health Screening Center, Kangbuk Samsung Hospital, Sungkyunkwan University School of Medicine, Seoul, Korea \\ ${ }^{3}$ Department of Family Medicine, Konkuk University Medical Center, Konkuk University School of Medicine, Seoul, Korea
}

Background: Several studies have revealed the frequency of family mealtimes to be inversely associated with depressive symptoms in adolescents. However, there have been few studies in older populations. This cross-sectional study investigated the association between family mealtime frequency and depressive symptoms in elderly Koreans.

Methods: This study analyzed 4,959 elderly men and women (aged 65 years or older) who participated in the Korea National Health and Nutrition Examination Survey. Self-administered questionnaires were used to assess depressive status, family mealtime frequency, and covariates. Multiple logistic regression analysis was performed to evaluate the association using the eating alone group as a reference.

Results: After adjusting for all covariates, participants who had family meals 3 times a day had fewer depressive symptoms than the eating alone group; adjusted odds ratios (ORs) (95\% confidence intervals [CIs]) were 0.72 (0.580.89 ) for point depressiveness/anxiety and $0.73(0.56-0.94)$ for depressiveness lasting for at least 2 weeks. In suicidal ideation, the OR ( $95 \% \mathrm{CI})$ of eating with family twice a day was significant after full adjusting for covariates at 0.67 (0.50-0.88).

Conclusion: Family mealtimes were closely associated with depressive symptoms in elderly Koreans, which suggests that maintaining intrafamilial bonding is important for mental health in an older population.

Keywords: Aged; Depression; Family Relations; Meals; Koreans

Received: May 11, 2017, Revised: August 1, 2017, Accepted: August 10, 2017

*Corresponding Author: Yun-Mi Song https://orcid.org/0000-0001-9232-5563

Tel: +82-2-3410-2449, Fax: +82-2-3410-0388, E-mail: yunmisong@skku.edu 


\section{INTRODUCTION}

Although the prevalence of late-life depression reported by several studies varies from lower than $1 \%$ to more than $35 \%,{ }^{1-3)}$ depression is one of the major health problems in the elderly, and the burden of inadequately treated late-life depression is substantial. ${ }^{2,4)}$ The World Health Organization predicted that depression would be the second greatest cause of disease burden by the year $2020,{ }^{5,6)}$ probably due to the remarkably accelerating rate of population aging. ${ }^{7)}$ Studies among elderly Koreans also found that the prevalence of overall late-life depression was higher than $10 \%$ and tended to increase with age. ${ }^{8)}$

Depression lowers quality of life and increases the risk of disability and morbidity ${ }^{6,9,10)}$ In addition, depression in the elderly frequently coexists with medical illnesses and may be a risk factor for other diseases such as dementia. ${ }^{11,12)}$ However, late-life depression is often overlooked during clinical evaluation of an older population because it is mistakenly assumed to be a normal response to aging, physical loss, or other life events. ${ }^{12-14)}$ Thus, prevention of the development of depression in the elderly by promoting healthy aging and modifying risk factors for late-life depression would be desirable to the older population.

Several factors associated with depression in the elderly have been suggested, such as sex, employment status, income level, smoking, alcohol consumption, and physical activities. ${ }^{3,11,12,15)}$ Among the many risk factors, loneliness, sometimes combined with other physical and mental problems, is found to be a major risk factor increasing depressive symptoms in the elderly. ${ }^{16)}$ Therefore, it is found that most of the elderly prefer remaining engaged in social interactions. ${ }^{16)}$

Family mealtimes are when family members sit down and enjoy meals together. During the family mealtime, people may have a good opportunity to interact and communicate with each other, thus increasing intimacy and emotional bonding among family members. ${ }^{17,18)}$ However, the busy personal lives of people living in the rapidly changing modern society have reduced the opportunity for family mealtimes over the last several decades, which may have a deteriorating influence on the emotional health of modern people. ${ }^{19,20)}$ Several studies on adolescents have revealed that frequency of family mealtimes was inversely associated with substance use, depression, and suicide involvement, even after controlling for family connectedness. ${ }^{19,20)}$ However, the effect of family mealtimes on depression in the elderly has not been extensively evaluated. ${ }^{21,22)}$

In this regard, we specifically explored the association between family mealtime frequency and depressive symptoms in elderly Koreans using the data from a nationally representative sample after controlling for a range of confounding variables. We hypothesized that the elderly who frequently share their mealtimes with family would have fewer depressive symptoms than those who have fewer family meals.

\section{METHODS}

\section{Study Participants}

This study used data from the fifth and sixth Korea National Health and Nutrition Examination Survey collected from 2010 to 2014 by the

Table 1. Characteristics of study participants

\begin{tabular}{|c|c|c|c|c|}
\hline Characteristic & Men $(n=2,148)$ & Women $(n=2,811)$ & All $(n=4,959)$ & P-value \\
\hline Age (y) & $72.3 \pm 0.15$ & $73.3 \pm 0.14$ & $72.9 \pm 0.10$ & $<0.001$ \\
\hline Married & $2,110(98.0)$ & $2,763(98.3)$ & $4,873(98.1)$ & 0.557 \\
\hline Employed & $887(41.5)$ & $547(19.7)$ & $1,434(28.8)$ & $<0.001$ \\
\hline Income & & & & 0.339 \\
\hline Low & $530(25.1)$ & $724(26.4)$ & $1,254(25.8)$ & \\
\hline Intermediate & $1,088(51.1)$ & $1,431(51.5)$ & $2,519(51.3)$ & \\
\hline High & $530(23.8)$ & $656(22.2)$ & $1,186(22.9)$ & \\
\hline Drinking & $1,210(56.5)$ & $487(17.6)$ & $1,697(33.8)$ & $<0.001$ \\
\hline Smoking & & & & $<0.001$ \\
\hline Never & $355(16.4)$ & $2,595(91.4)$ & $2,950(60.2)$ & \\
\hline Ex & $1,293(59.8)$ & $123(5.1)$ & $1,416(27.9)$ & \\
\hline Current & $500(23.8)$ & $93(3.5)$ & $593(11.9)$ & \\
\hline \multicolumn{5}{|l|}{ Comorbidities } \\
\hline Cardiovascular disease & $316(14.4)$ & $301(10.7)$ & 617 (12.2) & 0.001 \\
\hline Arthritis & $284(13.3)$ & $1,297(46.2)$ & $1,581(32.5)$ & $<0.001$ \\
\hline Asthma & $102(4.5)$ & $165(6.4)$ & $267(5.6)$ & 0.017 \\
\hline Family mealtime* (/d) & & & & $<0.001$ \\
\hline None & $274(13.1)$ & $928(33.5)$ & $1,202(25.0)$ & \\
\hline 1 Mealtime & $218(10.5)$ & $321(12.7)$ & $539(11.8)$ & \\
\hline 2 Mealtimes & $455(20.8)$ & $494(17.1)$ & $949(18.7)$ & \\
\hline 3 Mealtimes & $1,201(55.5)$ & $1,068(36.7)$ & $2,269(44.5)$ & \\
\hline Point depressiveness/anxiety ${ }^{\dagger}$ & $280(13.8)$ & $621(22.0)$ & 901 (18.6) & $<0.001$ \\
\hline Depressiveness that had lasted for at least 2 wk & $220(10.2)$ & $515(18.7)$ & $735(15.2)$ & $<0.001$ \\
\hline Ever had suicidal ideation during the last $1 \mathrm{y}$ & $275(12.9)$ & $629(23.4)$ & $904(19.0)$ & $<0.001$ \\
\hline
\end{tabular}

Values are presented as mean \pm standard deviation or number (\%). P-values were estimated by chi-square test and t-test.

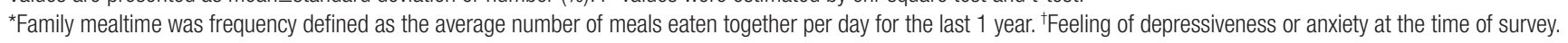


Korea Centers for Disease Control and Prevention. The Korea National Health and Nutrition Examination Survey was conducted among a representative sample of the Korean population, which was selected for the survey through a complex, multistage probability sample design on a nationwide basis. The survey consisted of a health survey, a nutrition survey, and medical examinations to provide statistics for evaluating public health policies. Among the 41,102 survey participants, we selected people who were aged 65 and older and had no missing values for all variables. Therefore, 4,959 people were included in this study. This study was reviewed and approved by the institutional review board of Samsung Medical Center (SMC 2016-04-008). The data used in this study do not include any identifiable personal identifiable personal information. Thus, the institutional review board exempted acquisition of informed consent form for the current study.

\section{Study Variables}

The main independent variable was family mealtime frequency per day during the past 1 year, which was assessed using the following question: "During the last year, did you usually have a meal with any family members? Please check for each meal: breakfast, lunch, or dinner." Using responses to the above question, we categorized study participants into four groups: 'eating alone,' 'eating with family once a day,' 'eating with family twice a day,' or 'eating with family 3 times a day'.

The dependent variables were depressive symptoms, which were assessed in three ways: point depressiveness/anxiety, depressiveness lasting for at least 2 weeks, and ever having had suicidal ideation during the last 1 year. For point depressiveness/anxiety, participants were categorized into two groups according to their responses to the self-assessment question, "How much do you feel depressed or anxious?" If a participant selected "I do not feel depressed or anxious," then he/she was categorized into the 'no point depressiveness/anxiety' group while one who selected other items ("I feel a little depressed or anxious" or "I extremely feel depressed or anxious") was categorized into the "point depressiveness/anxiety' group. Participants were assessed for depressiveness lasting for at least 2 weeks by the question "Have you been bothered by feeling sadness or hopelessness for 2 successive weeks in the last year?" The answers were categorized as "yes" or "no." Suicidal ideation was assessed by the question "Have you thought that you would be better off dead, or have you thought of hurting yourself in some way in the last year?" The answers were categorized as "yes" or "no."

We considered various sociodemographic and self-reported comorbidities as covariates given that these factors have been found to be associated with depression or quality of life in the elderly., ${ }^{3,12,18,21)}$ The sociodemographic variables comprised age, sex, marital status, employment status, income level, current alcohol consumption, and smoking behavior (i.e., never-smoker, former smoker, or current smoker). Current alcohol consumption was defined when participants drank alcohol at least once a month for the last year. Comorbid diseases comprised cardiovascular diseases including coronary artery disease and stroke, arthritis, and asthma.

\section{Statistical Analysis}

We performed a chi-square test for categorical variables and t-test for continuous variables to examine the differences in the distribution of each variable according to the presence of depressive symptoms. Multivariable-adjusted odds ratios (ORs) with $95 \%$ confidence intervals (CIs) were estimated by multiple logistic regression analysis to evaluate an independent association between depressive symptoms and family mealtime frequency after adjusting for covariates. The covariates were selected considering the findings of the univariate analysis in our study or previous studies ${ }^{3,12,18,21)}$ as follows: age, sex, marital status, employment status, and income level were adjusted in model 1. In model 2 , current alcohol consumption and smoking were additionally

Table 2. Association* of depressive symptoms with sociodemographic and clinical characteristics

\begin{tabular}{|c|c|c|c|c|c|c|}
\hline \multirow{2}{*}{ Variable } & \multicolumn{2}{|c|}{ Point depressiveness/anxiety } & \multicolumn{2}{|c|}{ Depressiveness lasting for at least 2 wk } & \multicolumn{2}{|c|}{ Ever had suicidal ideation during the last $1 \mathrm{y}$} \\
\hline & $\mathrm{OR}(95 \% \mathrm{Cl})$ & P-value & $\mathrm{OR}(95 \% \mathrm{Cl})$ & P-value & $\mathrm{OR}(95 \% \mathrm{Cl})$ & P-value \\
\hline Married & $0.52(0.36-0.77)$ & 0.001 & $0.45(0.27-0.76)$ & 0.002 & $0.62(0.37-1.01)$ & 0.056 \\
\hline Employed & $0.73(0.61-0.86)$ & $<0.001$ & $0.73(0.59-0.90)$ & 0.003 & $0.71(0.58-0.86)$ & 0.001 \\
\hline \multicolumn{7}{|l|}{ Income } \\
\hline Low & 1.00 & & 1.00 & & 1.00 & \\
\hline Intermediate & $0.75(0.63-0.88)$ & 0.001 & $0.70(0.57-0.86)$ & 0.001 & $0.82(0.68-0.99)$ & 0.035 \\
\hline High & $0.54(0.44-0.68)$ & $<0.001$ & $0.49(0.37-0.63)$ & $<0.001$ & $0.49(0.39-0.62)$ & $<0.001$ \\
\hline Drinking & $0.63(0.54-0.75)$ & $<0.001$ & $0.62(0.51-0.76)$ & $<0.001$ & $0.69(0.58-0.83)$ & $<0.001$ \\
\hline \multicolumn{7}{|l|}{ Smoking } \\
\hline Never & 1.00 & & 1.00 & & 1.00 & \\
\hline Ex & $0.63(0.53-0.75)$ & $<0.001$ & $0.56(0.45-0.70)$ & $<0.001$ & $0.68(0.55-0.83)$ & $<0.001$ \\
\hline Current & $0.66(0.52-0.85)$ & 0.001 & $0.87(0.67-1.12)$ & 0.277 & $1.05(0.83-1.34)$ & 0.696 \\
\hline Cardiovascular disease & $1.72(1.41-2.10)$ & $<0.001$ & $1.18(0.93-1.51)$ & 0.179 & $1.29(1.01-1.63)$ & 0.036 \\
\hline Arthritis & $1.91(1.64-2.22)$ & $<0.001$ & $1.77(1.48-2.12)$ & $<0.001$ & $1.70(1.43-2.02)$ & $<0.001$ \\
\hline Asthma & $1.84(1.39-2.44)$ & $<0.001$ & $1.20(0.82-1.75)$ & 0.349 & $1.48(1.05-2.10)$ & 0.026 \\
\hline
\end{tabular}

$\mathrm{OR}$, odds ratio; $\mathrm{Cl}$, confidence interval.

*Estimated by logistic regression analysis without adjustment for covariates. 
adjusted. In model 3, comorbidities with cardiovascular diseases, arthritis, and asthma were further adjusted.

Furthermore, we performed a stratified analysis to examine whether the association differed by sex; sex was not adjusted. The effect of sex on the association between depressive symptoms and family mealtime frequency was tested by including an interaction term (sex $\times$ family mealtime frequency) in the multiple logistic regression analysis. Statistical analyses were performed using PASW SPSS ver. 18.0 (SPSS Inc., Chicago, IL, USA). The statistical significance of each result was assessed based on $\mathrm{P}<0.05$.

\section{RESULTS}

Table 1 presents the demographic characteristics of study participants. The mean age was 72.3 years old for men and 73.3 years old for women. The prevalence of marital status and income level was similar between men and women. The prevalence of employment status, alcohol consumption, and smoking status was higher in men than in women. Among comorbidities, the prevalence of arthritis was significantly higher in women than in men. For family mealtimes, $75 \%$ of participants were found to have at least one family mealtime per day. The elderly who had less frequent family mealtimes $(\leq 1)$ were more commonly women $(46.2 \%)$ than men $(23.6 \%)$ while more frequent family mealtimes $(\geq 2)$ were more common in men $(76.3 \%)$ than in women $(53.8 \%)$. The prevalence of depressiveness was significantly higher in women than in men across all three variables assessing depressive symptoms.

Table 2 shows the association of depressive symptoms with sociodemographic and clinical characteristics. Sociodemographic variables except marital status and current smoking were inversely related with all three depressive variables. Among comorbidities, only arthritis was significantly associated with an increased risk of depressive symptoms across all three depressive variables.

Age-adjusted association between depressive symptoms and family mealtime frequency is presented in Table 3. Compared to eating alone, eating with family at least once a day was significantly associated with a decreased risk of depressive symptoms in all study participants.

Table 4 shows the multivariable adjusted association of depressive symptoms with family mealtime frequency. After full adjustment for covariates, participants who had family meals 3 times a day had fewer depressive symptoms than the eating alone group: adjusted ORs (95\% CIs) were $0.72(0.58-0.89)$ for point depressiveness/anxiety and 0.73 (0.56-0.94) for depressiveness lasting for at least 2 weeks. In suicidal ideation, the OR $(95 \% \mathrm{CI})$ of eating with family twice a day was significant after full adjusting for covariates at $0.67(0.50-0.88)$. In the analysis stratified by sex, participants having family meals 3 times a day showed a decreased risk of point depressiveness/anxiety after adjusting for all covariates regardless of sex; the OR (95\% CI) was 0.58 (0.39-0.88) for men and 0.76 (0.59-0.97) for women. However, the association between family mealtimes and two other depressive variables was significant only in men $(\mathrm{P}<0.05)$. There was no significant effect of sex on the association between depressive symptoms and family mealtime ( $\mathrm{P}$ interaction $>0.05$ ).

\section{DISCUSSION}

In this cross-sectional study conducted with a representative sample of elderly Koreans, we found that the frequency of family mealtimes was inversely associated with depressiveness as well as suicidal ideation, and the correlation was more evident in men than in women. This association persisted even after controlling for a range of sociodemographic factors and comorbid conditions. In addition, with increasing frequency of family mealtimes, ORs of point depressiveness/anxiety were decreased in a dose-response pattern.

The findings of our study are in accordance with those of previous studies, and they show that having regular family mealtimes serves as a protective factor against depressive mood, anger, and other psychological problems by enhancing family communication and building

Table 3. Age-adjusted association* between depressive symptoms and family mealtime frequency

\begin{tabular}{|c|c|c|c|c|c|c|c|}
\hline \multirow{2}{*}{ Variable } & \multirow{2}{*}{$\begin{array}{c}\text { Family } \\
\text { mealtime }(/ d)\end{array}$} & \multicolumn{2}{|c|}{ Point depressiveness/anxiety } & \multicolumn{2}{|c|}{ Depressiveness lasting for at least 2 wk } & \multicolumn{2}{|c|}{ Ever had suicidal ideation during the last $1 \mathrm{yr}$} \\
\hline & & OR $(95 \% \mathrm{Cl})$ & P-value & $\mathrm{OR}(95 \% \mathrm{Cl})$ & P-value & $\mathrm{OR}(95 \% \mathrm{Cl})$ & P-value \\
\hline \multirow[t]{4}{*}{ Men } & 1 Mealtime & $0.71(0.43-1.18)$ & 0.185 & $0.58(0.30-1.12)$ & 0.103 & $0.50(0.27-0.92)$ & 0.025 \\
\hline & 2 Mealtimes & $0.52(0.33-0.82)$ & 0.005 & $0.54(0.32-0.91)$ & 0.021 & $0.43(0.25-0.71)$ & 0.001 \\
\hline & 3 Mealtimes & $0.51(0.36-0.74)$ & $<0.001$ & $0.52(0.33-0.82)$ & 0.005 & $0.44(0.29-0.67)$ & $<0.001$ \\
\hline & P for trend & 0.003 & & 0.040 & & 0.001 & \\
\hline \multirow[t]{4}{*}{ Women } & 1 Mealtime & $0.79(0.57-1.10)$ & 0.168 & $0.81(0.57-1.16)$ & 0.244 & $0.99(0.69-1.42)$ & 0.953 \\
\hline & 2 Mealtimes & $0.64(0.48-0.86)$ & 0.003 & $0.68(0.48-0.96)$ & 0.028 & $0.62(0.45-0.84)$ & 0.002 \\
\hline & 3 Mealtimes & $0.72(0.57-0.91)$ & 0.007 & $0.68(0.51-0.89)$ & 0.006 & $0.80(0.62-1.01)$ & 0.063 \\
\hline & $P$ for trend & 0.019 & & 0.031 & & 0.011 & \\
\hline \multirow[t]{4}{*}{ All } & 1 Mealtime & $0.73(0.56-0.96)$ & 0.026 & $0.71(0.52-0.96)$ & 0.027 & $0.80(0.59-1.10)$ & 0.166 \\
\hline & 2 Mealtimes & $0.56(0.44-0.71)$ & $<0.001$ & $0.57(0.43-0.76)$ & $<0.001$ & $0.52(0.40-0.67)$ & $<0.001$ \\
\hline & 3 Mealtimes & $0.59(0.49-0.71)$ & $<0.001$ & $0.55(0.43-0.69)$ & $<0.001$ & $0.59(0.48-0.72)$ & $<0.001$ \\
\hline & P for trend & 0.000 & & 0.000 & & 0.000 & \\
\hline
\end{tabular}

$\mathrm{OR}$, odds ratio; $\mathrm{Cl}$, confidence interval.

${ }^{\star}$ Estimated by logistic regression analysis after adjusting for age. 


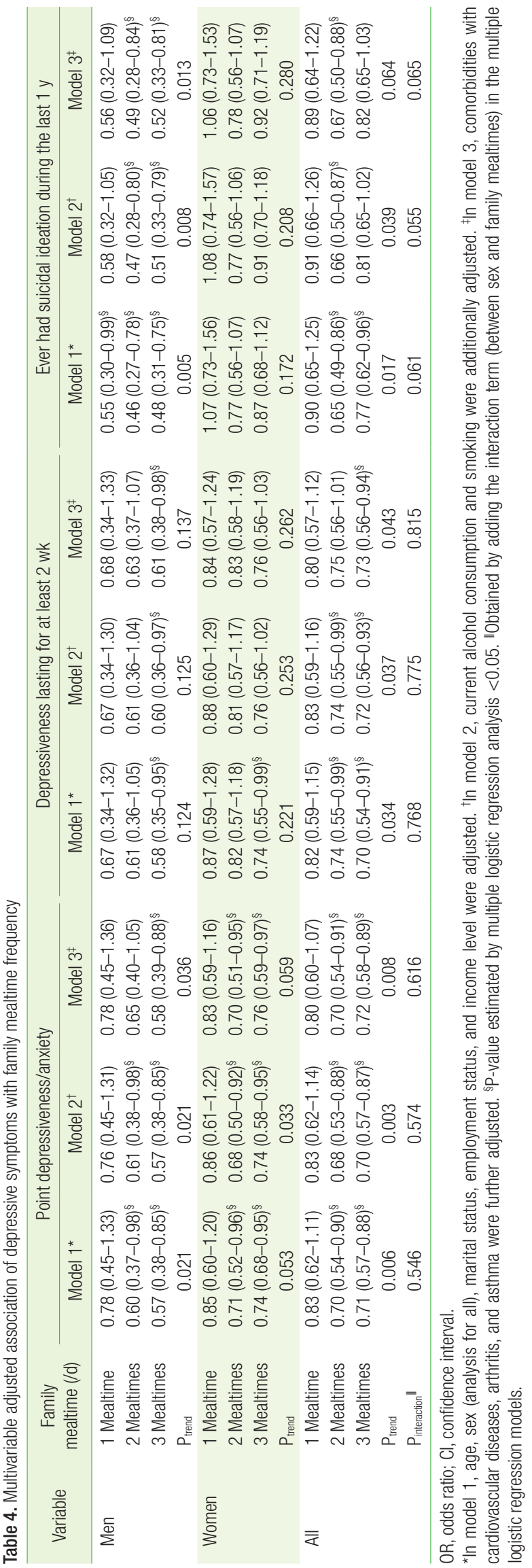

greater structure and stability in family relationships. ${ }^{17,19,23,24)}$ Family mealtimes, which allow family members to get together, communicate, and share emotions, could be helpful in alleviating negative effects of stressful experiences as well as depressive mood. ${ }^{14,17,22)}$ Family mealtimes on a regular basis also promote intimacy among family members by creating a sense of unity. ${ }^{17)}$ On the other hand, those who have meals alone tend to have relatively less interaction and lower social support compared to those who have meals with their family members. ${ }^{25)}$ The lack of communication and silence during mealtimes may result in feelings of loneliness and depressive mood. ${ }^{26)}$ Therefore, it may be necessary to emphasize the importance of participating in family mealtimes given the modern trend of living alone and the decreasing number of get-togethers within the family.

Interestingly, we found that elderly men were more likely to experience a beneficial effect on their mood by having family mealtimes compared to elderly women. According to previous studies on depression in men, sharing feelings or thoughts with others including family members has a more therapeutic effect on depression in men than in women. ${ }^{18,27)}$ In addition, having supportive relationships, such as discussing problems with trusted family members, contributes a lot to men's ability to cope with stress or depression. ${ }^{27)}$ Therefore, men who have meals alone may feel greater loneliness than those who usually have meals with their families, because they do not get an opportunity to discuss their stressful situations with family members.

In this study, the prevalence of having meals alone was $25.0 \%$. A previous study reported that the prevalence of having meals alone was $12.9 \%,{ }^{22)}$ which was much lower than in our study. Our study was conducted among elderly individuals aged 65 and older with a median age of 72.9 years old while the other study included participants younger than 65 years old (median age, 67 years old). ${ }^{22)}$ This difference in the age distribution of study participants could explain the discrepancy between the studies. Although further studies are necessary, we assume that the prevalence of having meals alone might increase with age. In our study, the frequency of family mealtimes was higher in men than in women, which was compatible with the finding of the other study. ${ }^{21)}$

The prevalence of depressive disorders in the elderly varies markedly across the studies, even in the same ethnic population. In our study, the prevalence of point depressiveness was $18.6 \%$, and the prevalence of depressiveness lasting for at least 2 weeks was $15.2 \%$. According to previous studies, the prevalence of major depression was reported to range from $4.6 \%$ to $9.3 \%$, and, for depressive disorder, the prevalence ranged from $4.5 \%$ to $37.4 \%{ }^{1,28)}$ Chong et al. ${ }^{29)}$ reported that the prevalence of depressive neurosis and major depression in the elderly in Taiwan was $15.3 \%$ and $5.9 \%$, respectively. Another study in Taiwan reported that the 1-month prevalence of clinically significant non-major depression in community-dwelling elderly (65 years of age or older) was $8.8 \%{ }^{30)}$ This variation in depression across studies could have resulted from varying cultural sensitivity to the diagnosis of depression, the psychometric tool, or innate biologic variation.

This study had certain strengths. First, we conducted this study us- 
ing a large representative sample of the Korean population. Second, we considered depressive mood in three ways: point depressiveness/ anxiety, depressiveness lasting for at least 2 weeks, and suicidal ideation during the last 1 year. In addition, we checked a wide range of covariates, including a number of clinical conditions that may have adverse effects on mood.

However, the findings of the present study should be viewed in light of several limitations. First, as it was a cross-sectional study, it was unable to explain a causal relationship between family mealtime frequency and depressive symptoms, and further longitudinal research is required. Second, we could not apply the diagnostic criteria for depressive disorder because the Korea National Health and Nutrition Examination Survey data lacked detailed questions needed for diagnosing clinical depression. Instead, we considered depressive symptoms in three ways including suicidal ideation to avoid hasty generalization. Third, we asked about depressiveness lasting for at least 2 weeks and suicidal ideation experienced during the last 1 year, which might have caused recall bias. Finally, we only assessed mealtimes with family because the Korea National Health and Nutrition Examination Survey data lacked detailed questions about mealtimes, so the eating alone group could include mealtimes with friends, which could cause underestimation in our conclusion. Despite these limitations, the present study provides significant outcomes as this was the first study to investigate the association between frequency of family meals and depressive symptoms in elderly Koreans.

In conclusion, this cross-sectional study revealed that family mealtimes were closely associated with depressive symptoms in elderly Koreans, which suggests that maintaining intrafamilial bonding is important for mental health in an older population.

\section{CONFLICT OF INTEREST}

No potential conflict of interest relevant to this article was reported.

\section{ACKNOWLEDGMENTS}

This research was supported by Basic Science Research Program through the National Research Foundation of Korea (NRF) funded by the Ministry of Science, ICT and future Planning (2014R1A2A2A010 02705).

\section{REFERENCES}

1. Beekman AT, Copeland JR, Prince MJ. Review of community prevalence of depression in later life. Br J Psychiatry 1999;174:307-11.

2. Battaglia A, Dubini A, Mannheimer R, Pancheri P. Depression in the Italian community: epidemiology and socio-economic implications. Int Clin Psychopharmacol 2004;19:135-42.

3. Bots S, Tijhuis M, Giampaoli S, Kromhout D, Nissinen A. Lifestyle- and diet-related factors in late-life depression: a 5-year follow-up of elderly European men: the FINE study. Int J Geriatr Psychiatry 2008;23:478-
84.

4. Lebowitz BD, Pearson JL, Schneider LS, Reynolds CF 3rd, Alexopoulos GS, Bruce ML, et al. Diagnosis and treatment of depression in late life: consensus statement update. JAMA 1997;278:1186-90.

5. Murray CJ, Lopez AD. Alternative projections of mortality and disability by cause 1990-2020: Global Burden of Disease Study. Lancet 1997;349:1498-504.

6. Mathers CD, Loncar D. Projections of global mortality and burden of disease from 2002 to 2030. PLoS Med 2006;3:e442.

7. Reddy MS. Depression: the disorder and the burden. Indian J Psychol Med 2010;32:1-2.

8. Park JH, Kim KW. A review of the epidemiology of depression in Korea. J Korean Med Assoc 2011;54:362-9.

9. Mecocci P, Cherubini A, Mariani E, Ruggiero C, Senin U. Depression in the elderly: new concepts and therapeutic approaches. Aging Clin Exp Res 2004;16:176-89.

10. Seymour J, Benning TB. Depression, cardiac mortality and all-cause mortality. Adv Psychiatr Treat 2009;15:107-13.

11. Kennedy GJ, Castro J, Chang M, Chauhan-James J, Fishman M. Psychiatric and medical comorbidity in the primary care geriatric patient: an update. Curr Psychiatry Rep 2016;18:62.

12. Raj A. Depression in the elderly: tailoring medical therapy to their special needs. Postgrad Med 2004;115:26-8,37-42.

13. Philippot $P$, Agrigoroaei S. Repetitive thinking, executive functioning, and depressive mood in the elderly. Aging Ment Health 2017;21:11926.

14. Rao SK, Wallace LMK, Theou O, Rockwood K. Is it better to be happy or not depressed?: depression mediates the effect of psychological well-being on adverse health outcomes in older adults. Int J Geriatr Psychiatry 2017;32:1000-8.

15. Fiske A, Wetherell JL, Gatz M. Depression in older adults. Annu Rev Clin Psychol 2009;5:363-89.

16. Singh A, Misra N. Loneliness, depression and sociability in old age. Ind Psychiatry J 2009;18:51-5.

17. Cason KL. Family mealtimes: more than just eating together. J Am Diet Assoc 2006;106:532-3.

18. Lee SA, Park EC, Ju YJ, Nam JY, Kim TH. Is one's usual dinner companion associated with greater odds of depression?: using data from the 2014 Korean National Health and Nutrition Examination Survey. Int J Soc Psychiatry 2016;62:560-8.

19. Eisenberg ME, Olson RE, Neumark-Sztainer D, Story M, Bearinger LH. Correlations between family meals and psychosocial well-being among adolescents. Arch Pediatr Adolesc Med 2004;158:792-6.

20. Neumark-Sztainer D, Hannan PJ, Story M, Croll J, Perry C. Family meal patterns: associations with sociodemographic characteristics and improved dietary intake among adolescents. J Am Diet Assoc 2003;103:317-22.

21. Jeong TW, Jung MJ, Lee JW, Kim TY. Effects of family meal frequency on risk factors for cardiovascular disease in Korean elderly males and females. Iran J Public Health 2015;44:60-7.

22. Wang X, Shen W, Wang C, Zhang X, Xiao Y, He F, et al. Association between eating alone and depressive symptom in elders: a cross-sectional study. BMC Geriatr 2016;16:19.

23. Goldfarb S, Tarver WL, Sen B. Family structure and risk behaviors: the role of the family meal in assessing likelihood of adolescent risk be- 
haviors. Psychol Res Behav Manag 2014;7:53-66.

24. Sen B. The relationship between frequency of family dinner and adolescent problem behaviors after adjusting for other family characteristics. J Adolesc 2010;33:187-96.

25. Vesnaver E, Keller HH. Social influences and eating behavior in later life: a review. J Nutr Gerontol Geriatr 2011;30:2-23.

26. Kimura Y, Wada T, Okumiya K, Ishimoto Y, Fukutomi E, Kasahara Y, et al. Eating alone among community-dwelling Japanese elderly: association with depression and food diversity. J Nutr Health Aging 2012;16:728-31.

27. Ramirez JL, Badger TA. Men navigating inward and outward through depression. Arch Psychiatr Nurs 2014;28:21-8.

28. Luppa M, Sikorski C, Luck T, Ehreke L, Konnopka A, Wiese B, et al. Age- and gender-specific prevalence of depression in latest-life: systematic review and meta-analysis. J Affect Disord 2012;136:212-21.

29. Chong MY, Tsang HY, Chen CS, Tang TC, Chen CC, Yeh TL, et al. Community study of depression in old age in Taiwan: prevalence, life events and socio-demographic correlates. Br J Psychiatry 2001;178:2935.

30. Chen CS, Chong MY, Tsang HY. Clinically significant non-major depression in a community-dwelling elderly population: epidemiological findings. Int J Geriatr Psychiatry 2007;22:557-62. 